**TITLE**

ASP Conference Series, Vol. **VOLUME**, **PUBLICATION YEAR **

**EDITORS ${ }^{* *}$

\title{
Radio Microlensing: Past, Present \& Near Future
}

\author{
L.V.E. Koopmans ${ }^{1,2,3}$, A.G. de Bruyn ${ }^{2,4}$, C.D. Fassnacht ${ }^{5}$, \\ J. Wambsganss ${ }^{6}$ \& R.D. Blandford ${ }^{1}+$ CLASS collaboration \\ ${ }^{1}$ Caltech, mail code 130-33, Pasadena 91125, US \\ ${ }^{2}$ Kapteyn Institute, P.O.Box 800, NL-9700 AV Groningen, Netherlands \\ ${ }^{3}$ JBO, Lower Withington, Macclesfield, Cheshire SK11 9DL, UK \\ ${ }^{4}$ NFRA-ASTRON, P.O.Box 2, NL-7990 AA Dwingeloo, Netherlands \\ ${ }^{5}$ NRAO, P.O.Box 0, Socorro, NM 87801, USA \\ ${ }^{6}$ U. of Potsdam, Inst. for Phys., Am Neuen Palais 10, 14469 Potsdam, \\ Germany
}

\begin{abstract}
Strongly correlated non-intrinsic variability between 5 and $8.5 \mathrm{GHz}$ has been observed in one of the lensed images of the gravitational lens B1600+434. These non-intrinsic (i.e. 'external') variations are interpreted as radio-microlensing of relativistic $\mu$ as-scale jet components in the source at a redshift of $z=1.59$ by massive compact objects in the halo of the edge-on disk lens galaxy at $z=0.41$. We shortly summarize these observations and discuss several new observational and theoretical programs to investigate this new phenomenon in more detail.
\end{abstract}

\section{Radio-Microlensing: B1600+434}

Multi-frequency observations of the CLASS gravitational lens (GL) B1600+434 with the VLA and WSRT radio telescopes at $1.4,5$ and $8.5 \mathrm{GHz}$ has unambiguously shown non-intrinsic variability in the lensed image that passes through the dark-matter halo of the edge-on disk lens galaxy (Koopmans \& de Bruyn 2000; Koopmans et al. 2000a). Based on the amplitude-timescale and frequency dependence of these non-intrinsic variations and the difference in variability between the two lensed images, separated by only 1 .' 4 , the non-intrinsic variability is interpreted as radio-microlensing of relativistic $\mu$ as-scale jet components in the source by massive compact objects in the halo of the edge-on disk lens galaxy. The alternatives, i.e. scintillation and extreme scattering, have considerable difficulties in explaining the time-scale and frequency dependence of the observations (e.g. Koopmans \& de Bruyn 2000; Koopmans et al. 2000a). An example of these variations at $5 \mathrm{GHz}$ is shown in Fig.1. The strong non-intrinsic event between days 70-100 is interpreted as a radio-microlensing caustic crossing. This type of event is extremely difficult to explain in terms of scattering (ISS) by the ionized interstellar medium (e.g. Koopmans et al. 2000b).

\section{Radio-Microlensing: Other Lens Systems \& Future}

To investigate radio-microlensing in B1600+434 and other lens systems in more detail, we have started a number of new monitoring programs with the WSRT, 


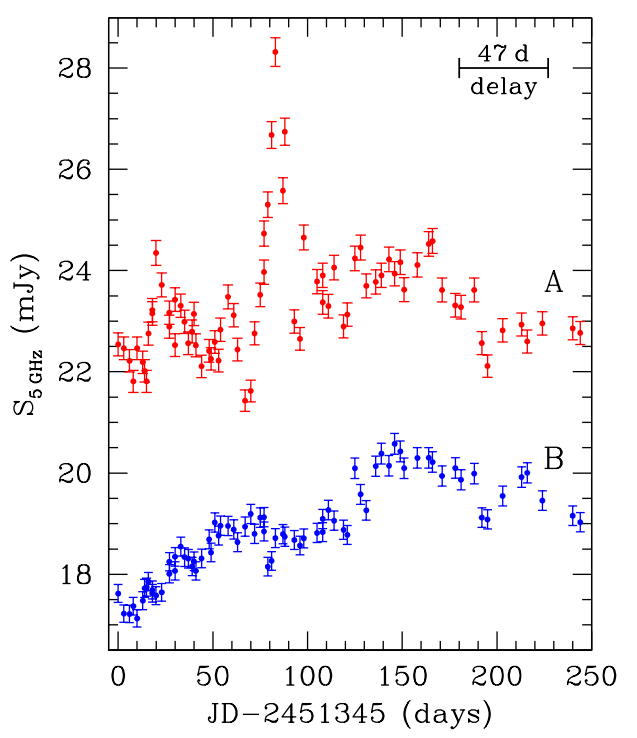

Figure 1. Preliminary results (at $5 \mathrm{GHz}$ ) from the 1999/2000 VLA monitoring campaign of $\mathrm{B} 1600+434$. The upper light curve (image A) passes through the darkmatter halo of the edge-on spiral lens galaxy. Note several strong (up to 30\%) events in the upper lightcurve and the complete absence of these events in the lower light curve (image B) after the measured time delay of $\sim 47$ days.

VLA and MERLIN radio telescopes. With the WSRT and VLA, we have been and are currently monitoring B1600+434 at 1.4, (1.7), 5.0, 8.5, 15 and (22) GHz. (Wavelengths between parentheses cover only part of the light curves.) This will enable us to further constrain the microlensing and ISS hypotheses based on their frequency dependence. In addition, we are currently monitoring 8 CLASS GL systems with the VLA at $8.5 \mathrm{GHz}$ to measure additional time-delays for the determination of $\mathrm{H}_{0}$. These lightcurves, however, also provide information on radio-microlensing. In addition, a MERLIN Key-Project to monitor 12 CLASS GL systems (2/3 quads) at $5 \mathrm{GHz}$ will conditionally start around Dec. 2000/Jan. 2001, with the aim of finding new cases of radio-microlensing. Combined, we will be able to search for this phenomenon in 14 aditional radio GL systems.

Besides this ongoing observational effort, we are theoretically studying radiomicrolensing in B1600+434, using numerical microlensing simulations, to place constraints on the fraction and mass-function of massive compact objects in the lens galaxy (Koopmans \& Wambsganss, in prep).

\section{References}

Koopmans, L.V.E \& de Bruyn, A.G., 2000, A\&A, 358, 793

Koopmans, L.V.E., de Bruyn, A.G., Wambsganss, J., Fassnacht, C.D., 2000a, to appear in Microlensing 2000: A New Era of Microlensing Astrophysics, eds J.W. Menzies and P.D. Sackett, ASP Conference Series

Koopmans, L.V.E., de Bruyn, A.G., Wambsganss, J., Fassnacht, C.D., Blandford, R.D., 2000b, to appear in "Cosmological Physics with Gravitational Lensing", Kneib J.-P., Mellier Y., Moniez M., Tran Thanh Van J. eds, Recontres de Moriond XX 